\title{
ESPRIT Based Robust Anti-Islanding Algorithm for Grid-Tied Inverter
}

Sandipan Patra

Technological University Dublin, Ireland, sandipan.patra@mydit.ie

Follow this and additional works at: https://arrow.tudublin.ie/ehsicon

Part of the Engineering Commons

\section{Recommended Citation}

Patra, S., Agrawal, S. \& Mohanty, S.R. ESPRIT Based Robust Anti-Islanding Algorithm for Grid-Tied Inverter. 2016 IEEE Students' Technology Symposium doi:10.1109/TechSym.2016.7872661

This Conference Paper is brought to you for free and open access by the ESHI Publications at ARROW@TU Dublin. It has been accepted for inclusion in Conference papers by an authorized administrator of ARROW@TU Dublin. For more information, please contact arrow.admin@tudublin.ie, aisling.coyne@tudublin.ie, gerard.connolly@tudublin.ie.

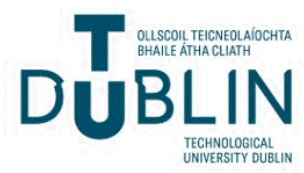




\title{
:三 Contents
}

OUBUIN INSTITUTE OF TECHNOLOCY INSTITIUID TECNEOLÁOCHTA ÁTHACLATH

muleso piuviueu ny.

DUBLIN INSTITUTE OF

TECHNOLOGY

Sign Out

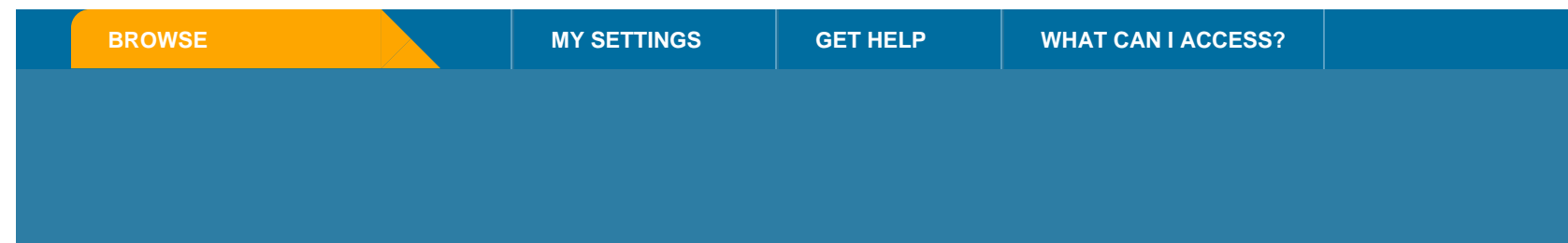

Basic Search

Author Search

Publication Search

Figures

Advanced Search

Other Search Options

References

Browse Conferences $>$ Technology Symposium $($ TechSym...

ESPRIT based robust anti-islanding algorithm for grid-tied inverter

Related Articles

Adaptive local polynomia varying frequency estimation

Keywords

Back to Top
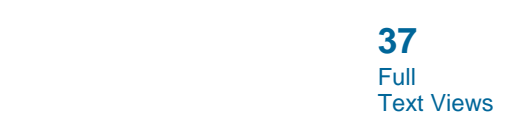

Operation of Single-phase Grid-Connected Inverters with Large DC Bus Voltage Rip...

Safe transient operation of microgrids based on master-slave configuration

View All

5

Author(s)
View All Authors

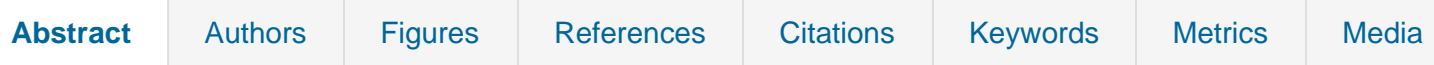

\begin{abstract}
:
This paper presents an anti-islanding protection scheme for a grid tied inverter. In this scheme, the oscillation variation in frequency is estimated by Estimation of Signal Parameters via Rotational Invariant Technique (ESPRIT). The proposed system is implemented in MATLAB and real time in OPAL-RT. Series of simulation results demonstrate that non-detection zone of proposed algorithm is negligible, and the scheme is also free from nuisance tripping to other transient events.
\end{abstract}

Published in: Technology Symposium (TechSym), 2016 IEEE Students'

Date of Conference: 30 Sept.-2 Oct. 2016

Date Added to IEEE Xplore: 09 March 2017

ISBN Information:

INSPEC Accession Number: 16726771

DOI: 10.1109/TechSym.2016.7872661

Publisher: IEEE

Conference Location: Kharagpur, India 
Download PDF

Download Citations

View References

Email

Print

Request Permissions

Export to Collabratec

Alerts

\section{SECTION I . Introduction}

Penetration of renewable energy/ distributed generation (DG) power resources such as the wind, solar photovoltaic (PV), solar thermal, wave energy into the conventional utility makes the operation, security, reliability, quality, and control problem profoundly challenging to power engineers and researchers [1]. Islanding is one of the significant protection issues, which still remains as a challenge. Islanding event occurs, when distributed energy source is disconnected from the utility grid and continues to energize the local load. This phenomenon can result in some potential hazards. Therefore, it is essential to detect islanding as soon as possible. According to IEEE 1547[2], islanding must be detected within 2 seconds.

Basic passive islanding protection schemes are over/ under-voltage protection (OVP/UVP) and over/ under frequency protection (OFP/UFP). These techniques are not able to detect islanding when power mismatch is small and also suffers from nuisance tripping to other transients [3].

Detailed literature survey of islanding detection techniques have been presented by Velasco et.al [3]. Islanding detection scheme can be classified into remote and local methods. Local methods are further classified mainly passive and active islanding detection scheme.

Active islanding scheme [4]- [5][6] usually injects perturbation signal into the system. It has no significant effect during parallel operation, but in the case of islanding event, these perturbation signal parameters become significant which makes active islanding detection more robust. Active islanding detection scheme have a small non-detection zone, but it deteriorates the power quality of power system during normal operating condition, and sometime maloperates due to the cancellation of the injected disturbance signal.

Passive islanding detection is based on the measurement of local parameters. A threshold value is set up for these parameters to discriminate islanding and non-islanding events. Passive islanding detection schemes have large non-detection zone (NDZ) [7]- [8][9]. Selection of most significant parameter and threshold value is a challenging task for developing passive islanding scheme. To decrease the NDZ of passive methods advanced signal processing methods are devised by the researchers [10]. In the present paper passive islanding detection approach is presented in which oscillation of frequency at the point of common coupling (PCC) is estimated. There are some signal processing tools to estimate oscillation frequency such as fast fourier transform (FFT), prony, and Estimation of Signal Parameters via Rotational Invariant Technique (ESPRIT) etc. Prony performance highly depends on the noise content of the signal which may lead to incorrect estimation [11]. FFT needs a large number of data points and also suffers from picket-fence leakage effect [12]. Thus, these techniques are not suitable for oscillation frequency estimation. In the proposed method, ESPRIT is used to estimate oscillation of frequency because its estimation accuracy is high and performance does not depend on noise content in the signal. It requires fewer data samples for estimation which makes implementation of the proposed scheme fast. This method will help to reduce the NDZ without any perturbation and does not affect output power quality. 


\section{SECTI ON II. \\ Proposed I slanding Detection Scheme}

In this section, proposed anti-islanding protection scheme is described. Behaviour of frequency pattern of voltage signal measured at PCC is different for islanding and non-islanding events. Frequency waveform of PCC voltage signal is shown in Fig. 1. Frequency profile of non-islanding events is oscillating in nature whereas islanding event frequency profile is monotonically increasing or decreasing. It means oscillation of frequency for islanding events is small as compared to the non-islanding event. In this paper, this particular property is used to develop antiislanding protection scheme.

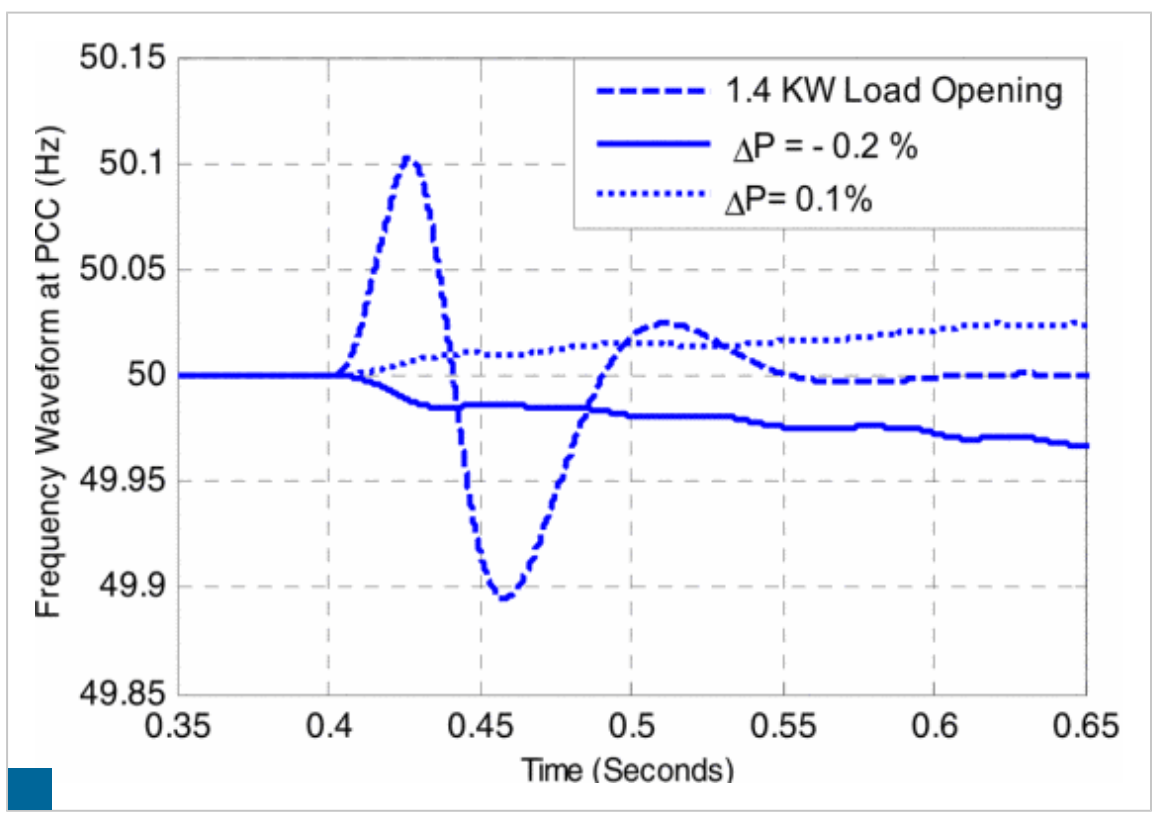

Fig 1.

Behavior of frequency at pcc

View All

A flow chart of the proposed algorithm is shown in Fig. 2. In this, the frequency of PCC signal is measured using phase locked loop (PLL) and sampled with sampling rate of $10 \mathrm{kHz}$. The difference between measured and nominal frequency ( $50 \mathrm{~Hz}$ ) is compared with a threshold $(0.012 \mathrm{~Hz})$ to determine whether the system is in normal operating condition or encountered any disturbance. If the difference between measured and nominal frequency crosses the threshold, it means the system is encountered by any disturbance, and it initiates data buffering. When buffered data samples are equal to 1000 samples, it goes to the oscillation frequency estimation block. ESPRIT function is embedded into MATLAB Simulink to estimate the oscillation frequency. Estimated oscillation frequency is compared with thresholds to discriminate the islanding and non-islanding events. These thresholds are determined by performing a number of simulations. Frequency oscillation for non-islanding disturbances (Load Switching, capacitor bank switching, distribution line faults, etc.) is high as compared to islanding events. Oscillation frequency for islanding event increases as power mismatch decreases and its maximum value $(5 \mathrm{~Hz})$ occurs when power mismatch is zero. Thus, islanding event occurs, when oscillation frequency is greater than zero and less than or equal to $5 \mathrm{~Hz}$. When estimated oscillation frequency satisfies this condition, islanding detection block disables the PWM signal, thus halting the inverter operation otherwise inverter is continued in operation and frequency difference is rechecked. 


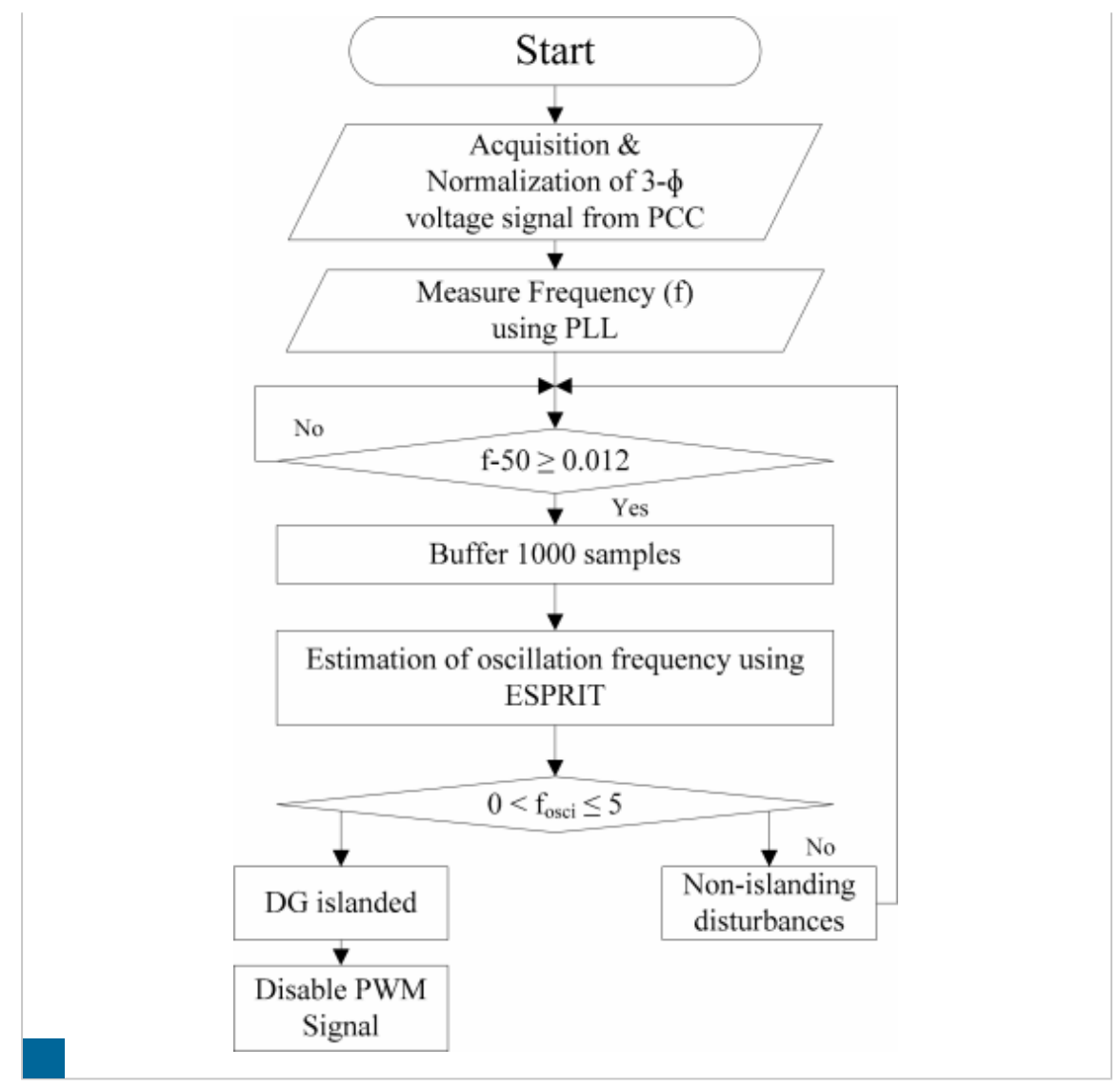

Fig. 2

Flow chart of proposed anti-islanding protection

View All

\section{SECTION III. Esprit}

ESPRIT is a parametric estimation technique that utilizes unique qualities of the autocorrelation matrix of a signal to estimate certain parameters of a signal that are possibly hidden in the noisy waveform. The implementation of the algorithm in power systems was proposed in [13], for estimation of harmonics. In power systems, the most common type of waveform encountered is one where a disturbance causes an oscillating transient that dies away with time. This can be modelled as a superposition of damped sinusoids in white noise as follows:

$$
x(n)=\sum_{i=1}^{M} A i e^{(\sigma i+j \omega i) n+J \phi i}+z(n)
$$

View Source?

Where $A_{i}$ is the initial amplitude, $\sigma_{i}$ is the damping factor, $\omega_{i}$ is the frequency $\phi_{i}$ is the initial phase, and $\mathrm{z}(\mathrm{n})$ is the noise component in the signal, reasonably modelled as white noise. These four quantities together define a damped sinusoidal mode. $M$ is the number of these modes in the signal. In other words, each estimated mode will be composed of a collection of these four quantities together. In the present method, it is used to estimate oscillation frequency. 


\section{SECTI ON I V. System Configuration}

A grid-connected PV inverter system is considered to prove the efficacy of the proposed signal processing techniques. The system configuration as shown in fig. 3 has been simulated on Matlab/ Simulink platform. Further, the real time version is developed in OPAL-RT.

The inverter is connected to the low-voltage side, which presents a grid of $75 \mathrm{~V} \mathrm{rms}$. Circuit breaker, SW1 is closed to connect the synchronised PV inverter to the grid, while circuit breaker is opened to experience a grid failure (islanding). The RMS of the three grid voltages, along with the PV inverter ac current reference (Iref), dc link voltage (Vdc), modulation index and value of coupling impedance of the inductor-capacitor-inductor (LCL) filter are used to calculate the required inverter peak voltage and lead angle. Vdc is sensed simultaneously. The positive sequence component is derived from a phase lock loop (PLL). The three-phase discrete PLL is then used to retrieve the frequency and phase information $(\omega \mathrm{t})$ of these normalised grid voltage signals. These information are used to generate the $3-\varphi$ reference signal for $20 \mathrm{kHz}$ PWM pulses. A $1.4 \mathrm{~kW}$ load is also connected through another circuit breaker (sw2) and it is opened to experience the nonislanding event like load switching.

After creating the islanding situation and to resolve the problem of NDZ for the passive islanding detection methods, ESPRIT techniques has been adopted. The frequency of the system has been retired from the PLL block and used to determine the islanding situation.

\section{SECTION $V$. Result and Discussion}

To show the efficacy of proposed algorithm, three case studies are presented in this paper.

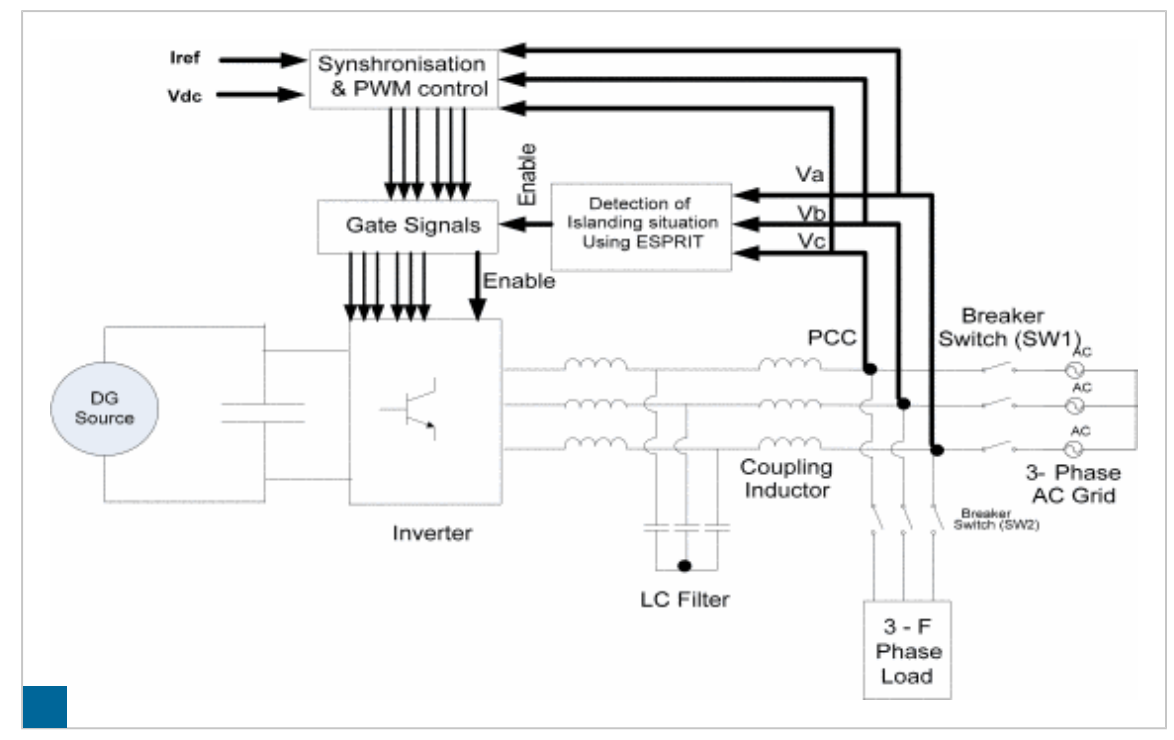

Fig. 3

System diagram for analysis 
1. Islanding event of large power mismatch: In this case local load demand is $1.55 \mathrm{~kW}$. Inverter supplies $1.4 \mathrm{~kW}$ and rest power demand met by the grid. An islanding event is created at the instant of 0.41 second by opening the circuit breaker. This leads to a large power mismatch (>10\%). In this case, frequency deviation at PCC is large.

2. Islanding event of small power mismatch: In this case local load demand is $1.402 \mathrm{~kW}$. Inverter supplies $1.4 \mathrm{~kW}$ and rest of the power demand is met by the grid. An islanding event is created at the instant of 0.41 second by opening the circuit breaker. This leads to a negligible power mismatch ( $0.2 \%)$. In this case, frequency deviation at PCC is very small.

3. Non-islanding event (load switching): In this case local load of $1.4 \mathrm{~kW}$ is disconnected at the instant of 0.41 second. This results in a disturbance. In this case, frequency deviation at PCC also occurs

\section{A. Over Frequency and Under Frequency Protection (ofp/ufp)}

First, the performance of conventional OFP/ UFP is determined for case 1 and case 2. OFP and UFP relay operating point is defined as 50.2 and 49.8 respectively and it is shown by dotted lines in Fig. 4 and Fig. 5. When PCC frequency crosses beyond these boundary values, relay will operate. In the case of large power mismatch, Frequency deviation is large and it crosses the operating point and detects islanding event within two cycles as shown in Fig. 4. When power mismatch is small, frequency deviation is very small and it lies within the boundary values as shown in Fig. 5 and the relay fails to detect islanding event. OFP/ UFP suffers from large NDZ and also can cause maloperation to disturbance other than non-islanding event.

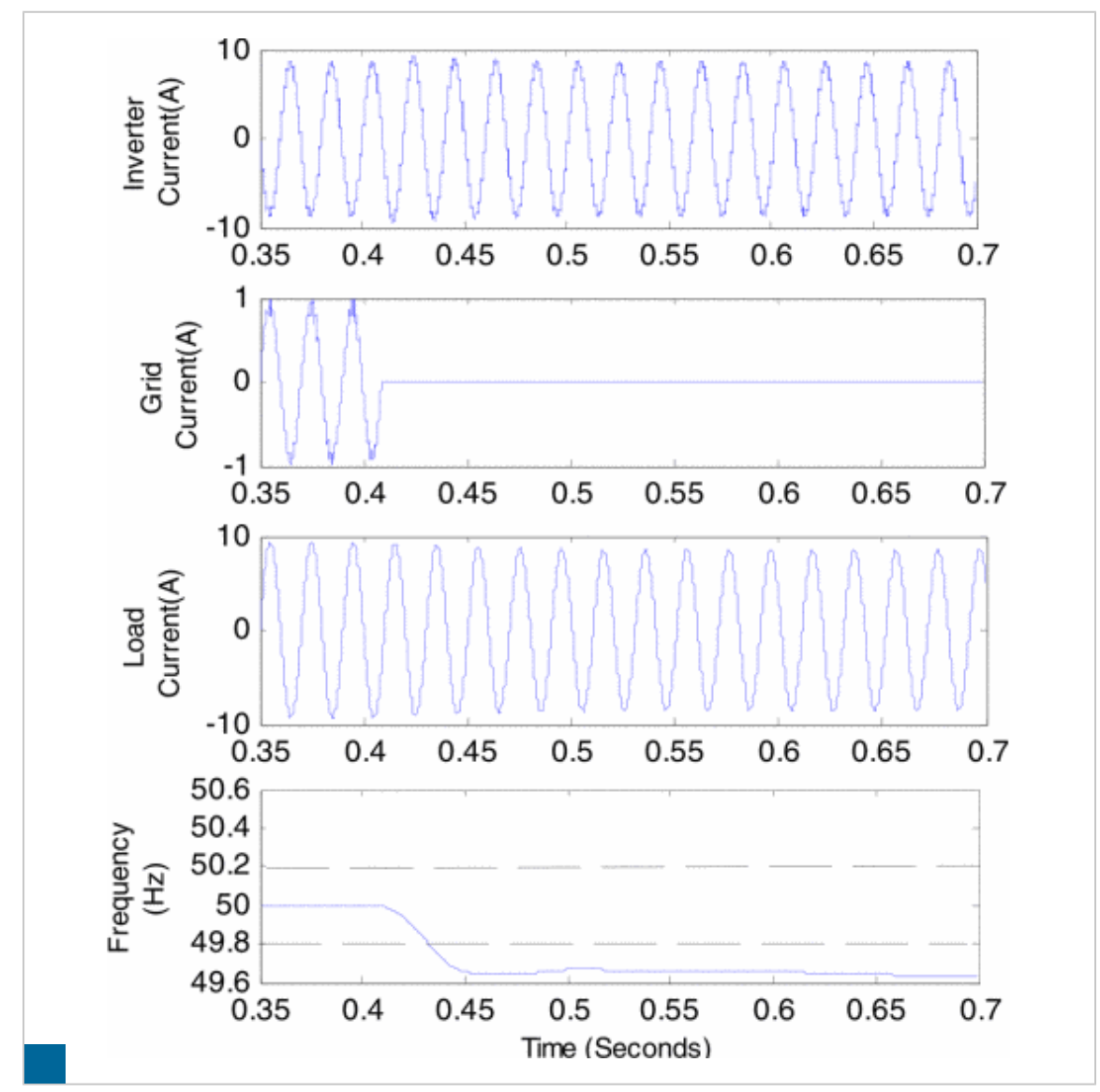

Fig. 4

Case 1 of large power mismatch with ofp/ufp method 


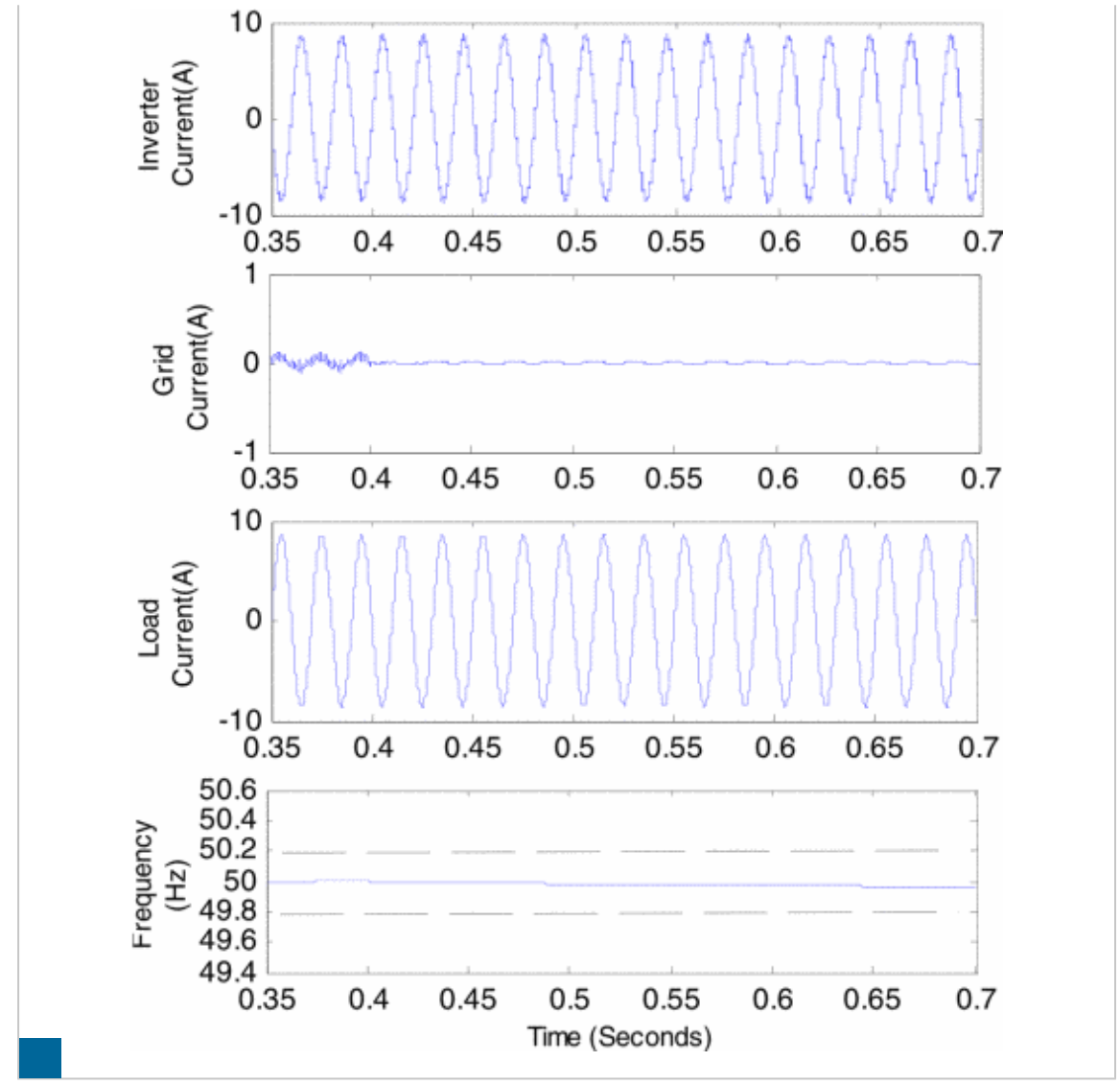

Fig. 5

Case 2 of small power mismatch with ofp/ufp method

View All

\section{B. Proposed Method}

Performance of the proposed anti-islanding protection method is verified in both Simulink and OPAL-RT. This section is divided into two parts. In the first part simulation results for all three cases are presented and realtime software-in-the-loop (SIL) results are demonstrated in the second part.

\section{1) Simulation Results}

Simulation results for case 1, case2 and case3 are shown in Fig. 6, 7, and 8 respectively. In Case1 of large power mismatch islanding event, estimated oscillation frequency is $4 \mathrm{~Hz}$ and islanding event is detected at 0.6 second. Proposed islanding protection method takes 0.2 seconds time to disable the PWM signal. It takes 0.1 second for data buffering and 0.1 second for computation. Total protection time of the proposed method is much less than the IEEE1547 specified time limit of 2 seconds.

In case 2 of small power mismatch islanding event, estimated oscillation frequency is close to $5 \mathrm{~Hz}$ and it detects islanding event at 0.6 second. It ensures that proposed method detection time is independent of power mismatch and it detects islanding event even when the power mismatch is negligible.

Load of $1.4 \mathrm{~kW}$ is opened in Case 3, which means, it is a non-islanding event. Estimated oscillation frequency is greater than the $5 \mathrm{~Hz}$ and it does not disable the PWM signal. It signifies that proposed algorithm is free from mal-operation. 


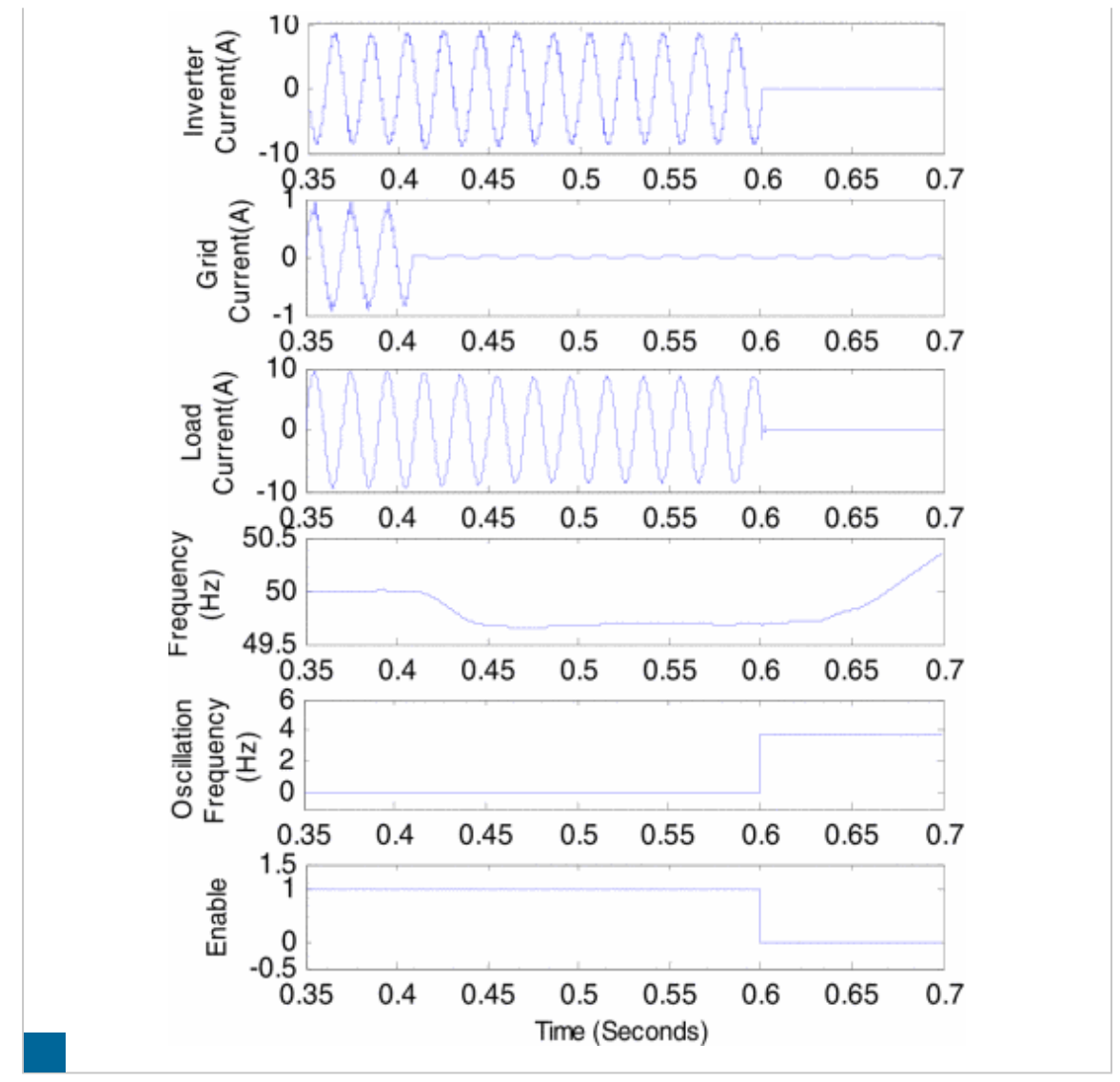

Fig. 6

Case 1 of large power mismatch (ndz) with proposed protection scheme
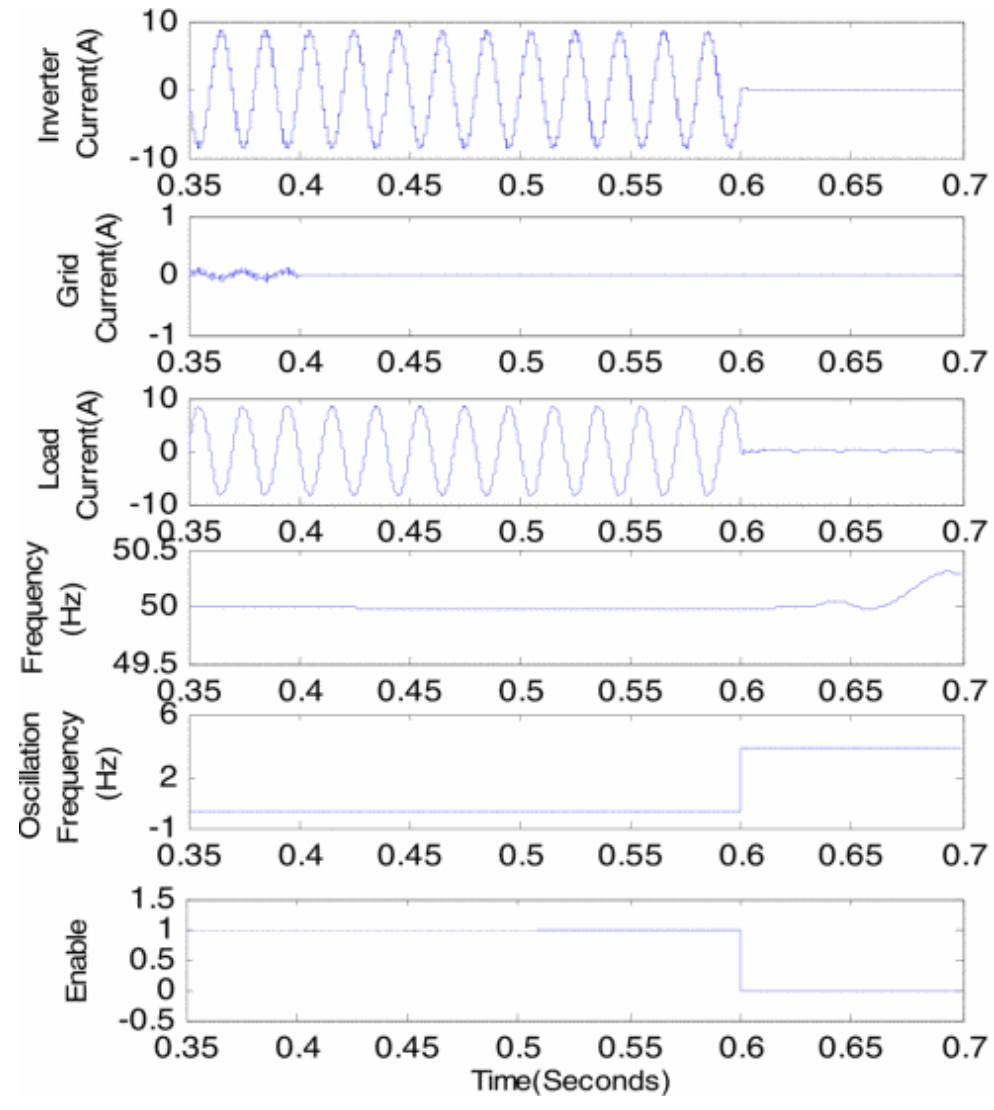

Fig. 7 


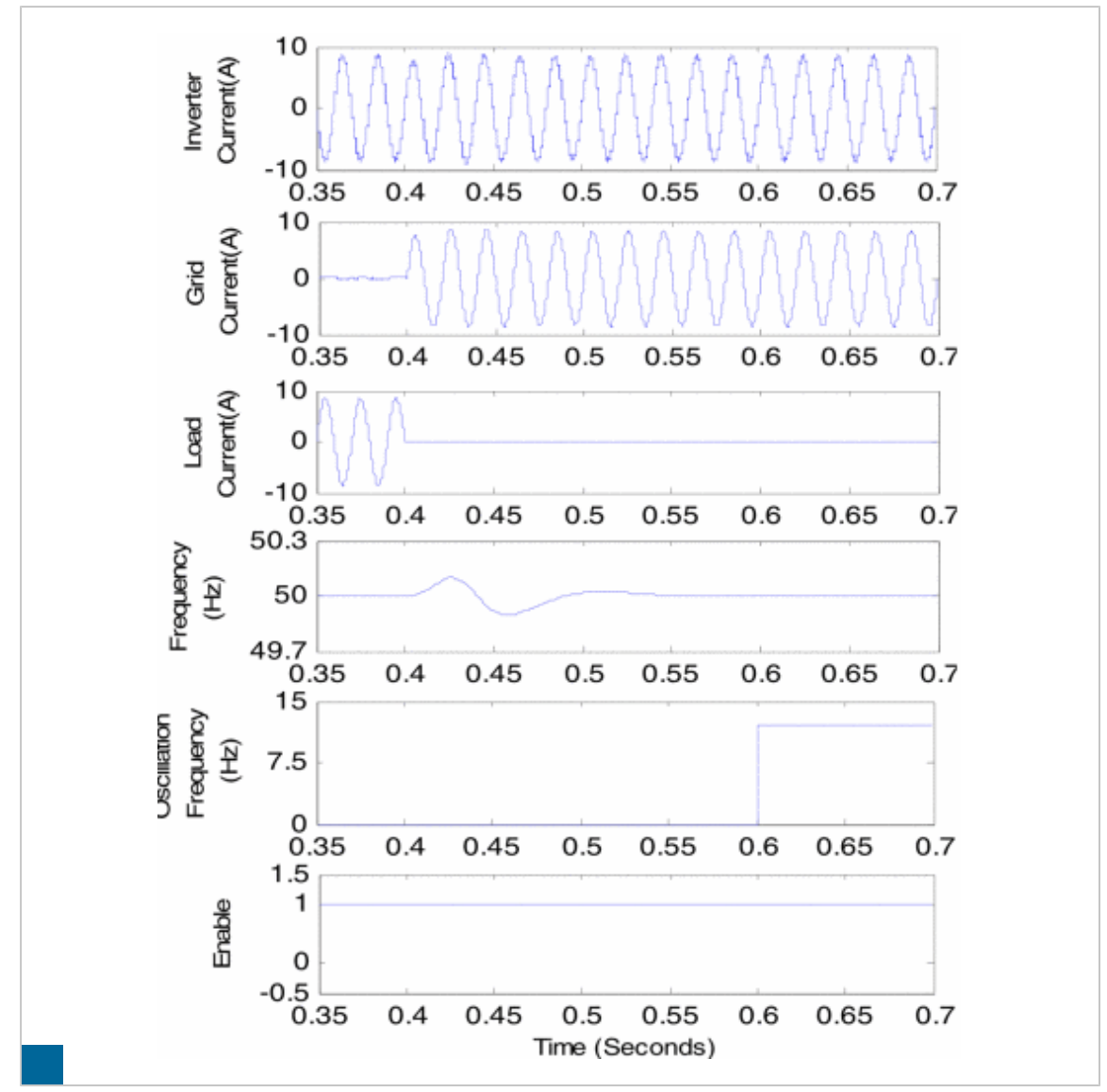

Fig. 8

Case 3 of load switching (non-islanding event) with proposed protection scheme

\section{2) Real-Time Software-in-the-ioop(sil) Results}

The proposed anti-islanding protection method along with the connected grid-tied inverter system have been modelled in Simulink by using the RT-LAB real-time platform and the associated tools. It provides platform to check the performance of proposed method in a real-time environment. The system is then tested in the software-in-the-loop (SIL) in the hardware synchronization mode, which is similar to the hardware-in-the-loop (HIL) test giving due consideration to the delay in the realtime measurement of the actual signals. All the real time results are obtained from the OP5600 OPAL-RT and the amplitude of all the signals are scaled down by 10 times.

All three cases are verified in real-time. Fig. 9 and 10 shows the real-time result of the proposed scheme for case $1 \& 2$ respectively. It is observed that proposed anti-islanding protection scheme works satisfactorily and detection time in real-time is 0.1 , which is half of the time taken in simulation. Its computation time in real-time is negligible which makes the proposed scheme more effective. Fig. 11 shows the real time result of the case 3 where load switching occurs. As it is not an islanding event so the enable signal remains constant. And the inverter current is equal to grid current after disconnecting the load.

From the Fig. 9, 10 and 11, it is clearly observed that proposed anti-islanding scheme detects islanding event efficiently even for negligible power mismatch and does not operate at nonislanding disturbance events. 


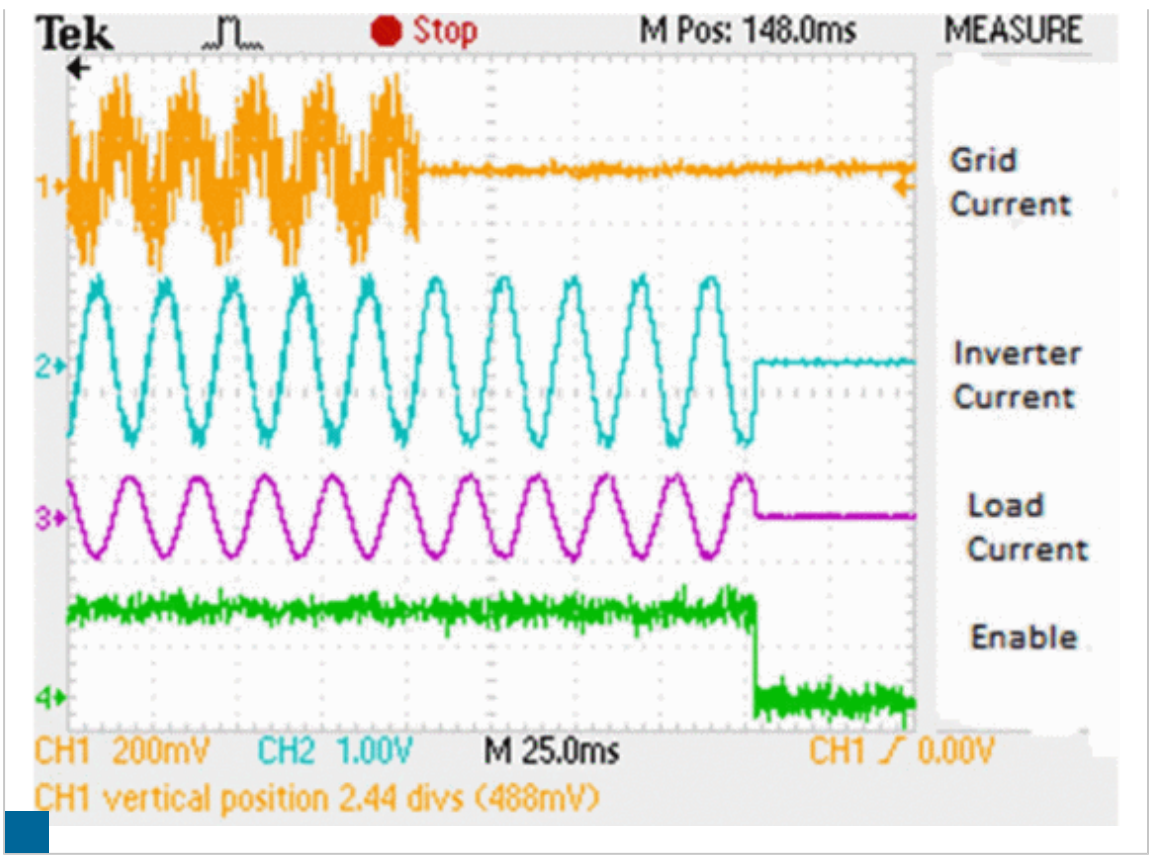

Fig. 9

Case 1 of large power mismatch (ndz) with proposed protection scheme in real time.

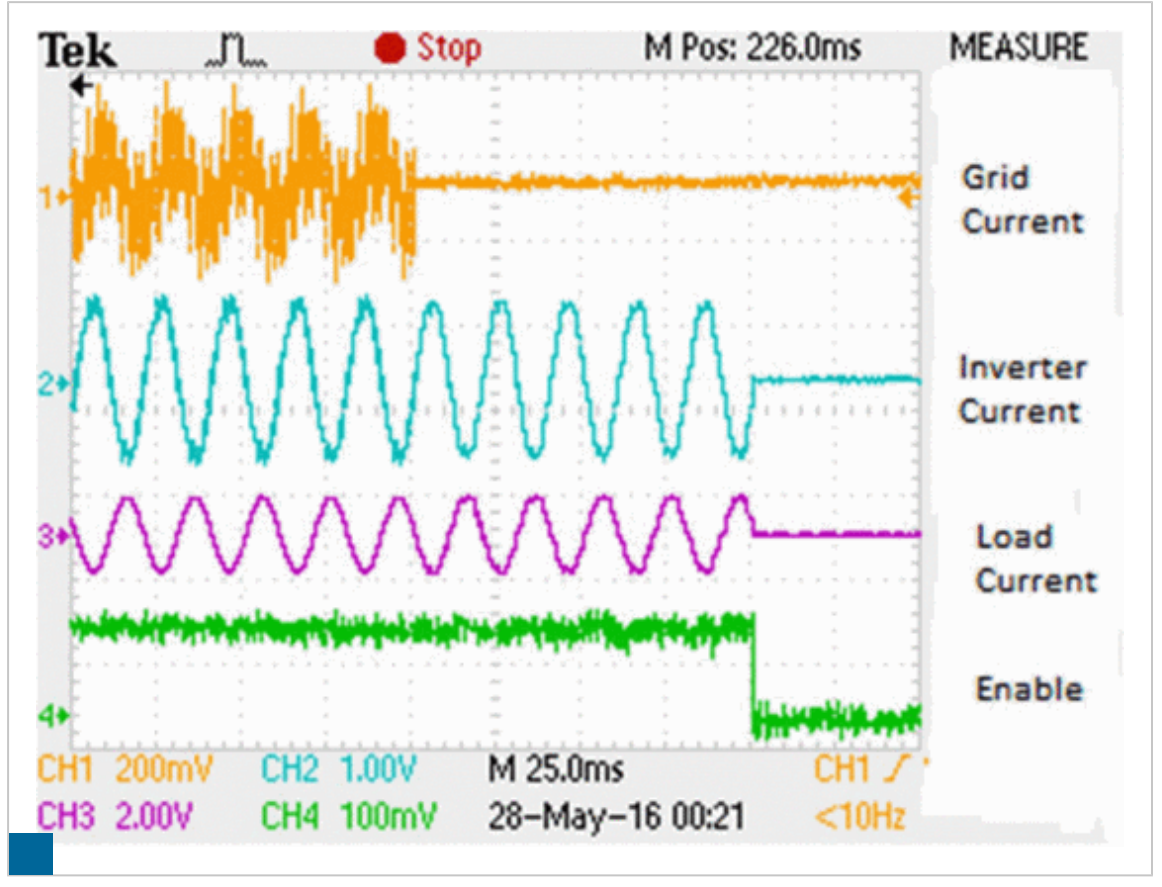

Fig. 10

Case 2 of small power mismatch (ndz) with proposed protection scheme in real time. 


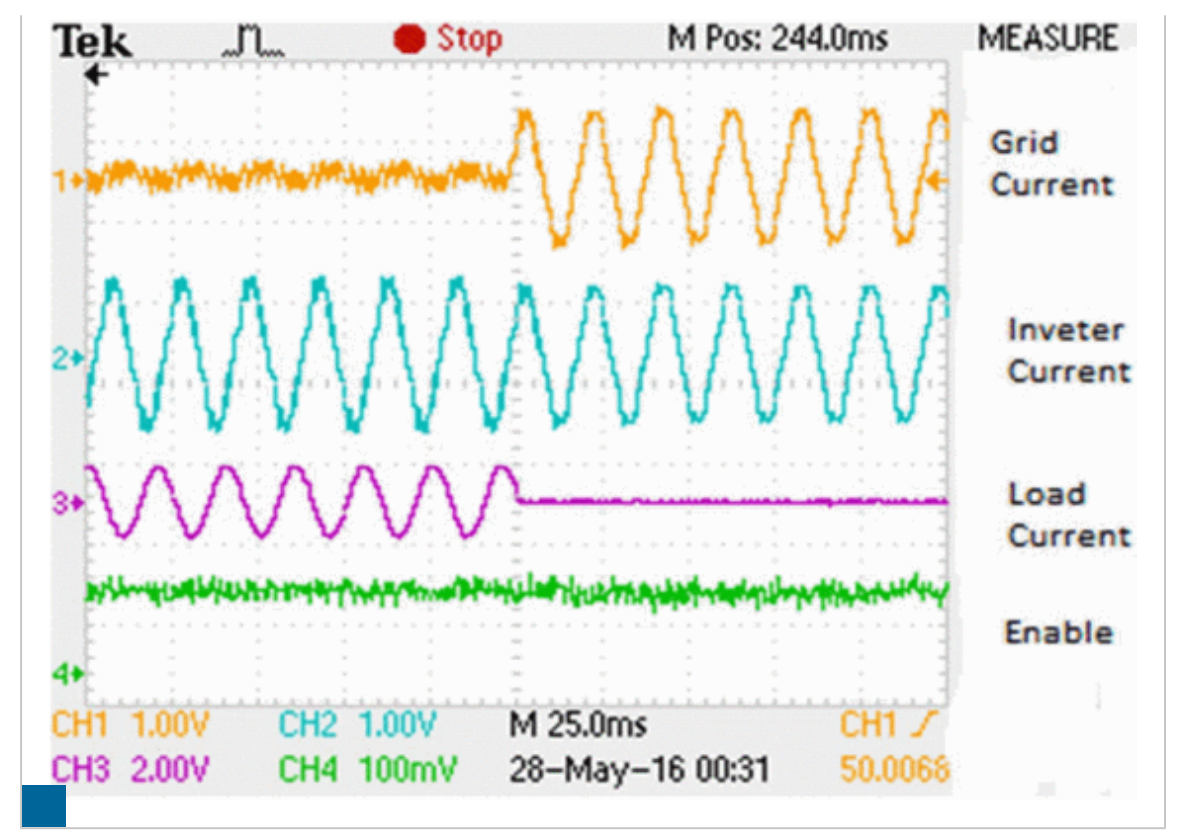

Fig. 11

Case 3 of load switching (non-islanding event) with proposed protection scheme in real time

View All

\section{SECTION VI . Conclusion}

Proposed ESPRIT based anti-islanding scheme is found to be very effective in discriminating between islanding detection and other disturbances. NDZ is very small in the proposed scheme and it avoids nuisance tripping because of other transients. Detection time of proposed detection method is also small. Islanding detection performance is measured in terms of detection time and non-detection zone. Series of simulation and real-time results prove its effectiveness in both the measures.

\section{ACKNOWLEDGEMENT}

This work has been carried out with the support of Science Foundation Ireland - International Strategic Cooperation Award Programme (SFI-ISCA). The real time implementation is performed in Dublin Institute of Technology, Ireland.

\section{Keywords}

\section{IEEE Keywords}

Oscillators, Inverters, Frequency estimation, Islanding, Time-frequency analysis, Estimation

\section{INSPEC: Controlled Indexing}

distributed power generation, frequency estimation, invertors, oscillations, power generation

protection, power grids, power system simulation 


\section{INSPEC: Non-Controlled Indexing}

ESPRIT based robust anti-islanding algorithm, grid-tied inverter, anti-islanding protection, oscillation variation, frequency Estimation, estimation of signal parameter via rotational invariant technique, MATLAB, OPAL-RT, nondetection zone, nuisance tripping, transient events

\section{Author Keywords}

Anti-islanding Schemes, Grid-tied Inverter, ESPRIT, Non-Detection Zone

\section{Authors}

Sandipan Patra

School of Electrical \& Electronic Engineering, Dublin Institute of Technology

Sanjay Agrawal

Department of Electrical Engineering, MNNIT, Allahabad

Soumya R Mohanty

Department of Electrical Engineering, MNNIT, Allahabad

Vineeta Agarwal

Department of Electrical Engineering, MNNIT, Allahabad

Malabika Basu

School of Electrical \& Electronic Engineering, Dublin Institute of Technology

\section{Related Articles}

Adaptive local polynomial periodogram for time-varying frequency estimation

V. Katkovnik

Operation of Single-phase Grid-Connected Inverters with Large DC Bus Voltage Ripple Nayeem A. Ninad; Luiz A. C. Lopes

Safe transient operation of microgrids based on master-slave configuration

Gustavo M. S. Azevedo; Fabricio Bradaschia; Marcelo C. Cavalcanti; Francisco A. S. Neves; Joan Rocabert; Pedro Rodriguez

Definitions of "total" estimators of common time-domain variances

D.A. Howe; T.K. Peppler

FPGA-based implementation of an adaptive notch filter used for grid synchronization of gridconnected converters Matthew Mascioli; Majid Pahlevaninezhad; Praveen Jain

Fundamental limits on phase and frequency tracking and estimation in drifting oscillators D. R. Brown; R. Mudumbai; S. Dasgupta

FPGA implementation of fixed and variable frequency ADALINE schemes for grid-connected VSI synchronization

Alben Cardenas; Cristina Guzman; Kodjo Agbossou 
Use of the cross polynomial Wigner-Ville distribution for instantaneous frequency estimation of non-linear FM signals

B. Ristic; B. Boashash

Grid Monitoring for Distributed Power Generation Systems to Comply with Grid Codes Adrian V. Timbus; Remus Teodorescu; Frede Blaabjerg; Pedro Rodriguez

Carrier frequency offset estimation approach for multicarrier transmission on hexagonal timefrequency lattice Kui Xu; Wenfeng Ma; Lianguo Wu; Wei Xie; Dongmei Zhang; Youyun Xu

\section{IEEE Account}

»Change Username/Password

»Update Address

$\begin{array}{ll}\text { Purchase Details } & \text { Profile Information } \\ \text { »Payment Options } & \text { »Communications Preferences } \\ \text { »Order History } & \text { »Profession and Education } \\ \text { »View Purchased Documents } & \text { »Technical Interests }\end{array}$

Need Help?

US \& Canada: +18006784333

"Worldwide: +1 7329810060

»Contact \& Support

About IEEE Xplore | Contact Us | Help | Terms of Use | Nondiscrimination Policy | Sitemap | Privacy \& Opting Out of Cookies 